\title{
HEPATOBILIARY SCANNING STANDARDS FAIL TO IDENTIFY SYMPTOMATIC PATIENTS WITH CHRONIC ACALCULOUS CHOLECYSTITIS
}

\author{
Original Clinical Research
}

Matthew D. Stanley, BS; Tiffany N. Wright, MD; Daniel L. Davenport, PhD;

J. Scott Roth, MD; Andrew C. Bernard, MD

Division of General Surgery, Department of Surgery, University of Kentucky Medical Center, Lexington, $\mathrm{KY}$

Address Correspondence and Reprint Requests to:

Andrew C. Bernard, M.D.

C206 Division of General Surgery UK College of Medicine

800 Rose Street

Lexington, Kentucky 40536-0298

859-323-6346 Ext 224; 859-323-6840 (Fax)

acbern00@uky.edu 


\section{ABSTRACT}

Background: Patients with chronic biliary colic-like pain and normal transabdominal ultrasonography (TUS) typically undergo nuclear hepatobiliary imaging (HIDA) with cholecystokinin (CCK) injection and gall bladder ejection fraction (EF) calculation to identify gallbladder dyskinesia. Most literature suggests $\mathrm{EF} \leq 35 \%$ indicates biliary dyskinesia. We hypothesize that presence of chronic biliary colic-like symptoms predicts favorable outcome after cholecystectomy (CCY) in patients with normal hepatobiliary imaging.

Methods: A retrospective review of 1295 patients who underwent laparoscopic CCY from January 2006 to December 2010 in a tertiary care institution was completed. Patients with gallstones on TUS or diagnostically abnormal HIDA scans were excluded. Symptom reproduction upon $\mathrm{CCK}$ injection was not considered for inclusion criteria but was noted upon chart review. Patient outcome, favorable or unfavorable, was determined from postoperative follow-up.

Results: One hundred and six patients (8.19\%) with normal TUS and HIDA scans were included in the final analysis. Ninety-six patients $(90.6 \%)$ reported a favorable outcome (i.e., significant improvement or complete resolution of their symptoms) on follow-up.

Ninety-three patients with favorable outcomes and six patients with unfavorable outcomes presented with an initial complaint of right upper quadrant (RUQ) and/or epigastric pain $(\mathrm{p}=0.001$, sensitivity=95.9\%, PPV=93.9\%). Sixty-eight patients with favorable outcomes and one patient with an unfavorable outcome presented with an initial complaint of postprandial RUQ and/or epigastric pain ( $\mathrm{p}<0.001$, specificity $=90.0 \%, \mathrm{PPV}=98.6 \%$ ).

Conclusion: Hepatobiliary imaging fails to identify all patients suffering from chronic acalculous cholecystitis. Presence of chronic biliary colic-like symptoms predicts benefit from CCY 


\subsection{INTRODUCTION}

Chronic acalculous cholecystitis $(\mathrm{CAC})$ is a functional hepatobiliary disease of poorly understood etiology and increasing national prevalence (Barbara, et al., 1987; Adams, et al., 1998; Johanning \& Gruenberg, 1998). For physicians, it is a controversial and indeterminate diagnosis of exclusion requiring elimination of other diseases (i.e., irritable bowel syndrome, cystic duct spasm, sphincter of oddi dysfunction, and gastroesophageal reflux) with similar presentation (i.e., right upper quadrant (RUQ), postprandial pain with nausea and/or vomiting) and pathophysiology. Transabdominal ultrasonography (TUS) to assess for gallstones is the standard of care for patients with chronic biliary-like pain. Normal TUS with persistent symptomatology precipitates a hepatobiliary immunodiacetic acid (HIDA) scan and gallbladder ejection fraction (EF) calculation to assess for biliary dyskinesia. TUS and HIDA scans with EF calculation are effective, non-invasive techniques for identifying patients that will benefit from cholecystectomy (CCY) due to the presence of identifiable gallstones or biliary dyskinesis (Fink-Bennett, et al., 1991; Yap, et al., 1991; Ross, et al., 2011).

Normal TUS and HIDA with EF studies present a dilemma that can delay diagnosis while additional symptom etiologies are explored. Published studies estimate that $5 \%$ to $25 \%$ of patients with pathologic evidence of chronic cholecystitis have normal TUS (Gracie \& Ransohoff, 1992; Chen, et al., 2001). No published studies provide data about the number of patients with normal HIDA scans and pathologic evidence chronic cholecystitis. In fact, controversy surrounds the distinction between normal and abnormal EF values. The majority of published studies suggest delineation is at approximately 35\% (FinkBennett, et al., 1991; Yap, et al., 1991; Chen, et al., 2001; Davis, et al., 1982; Topper, et al., 1980; Pickleman, et al., 1985; FinkBennett, et al., 1985; Misra, et al., 1991; Zech, et al., 1991; Barron, et al., 1995; Yost, et al., 1999; Middleton \& Williams, 1999; Poynter, et al., 2002; Majeski, et al., 2003).

The authors observed patients at our institution presenting with chronic biliarylike pain symptoms, normal TUS, and normal HIDA scan EF, who benefited from CCY. Pathology demonstrated CAC in the majority of these patients.

To the best of our knowledge, only two published studies analyzed outcome after CCY in patients with normal (i.e., $>35 \%$ ) gallbladder EF. Fuller and colleagues identified 55 patients with both normal $(\mathrm{N}=33)$ or abnormal $(\mathrm{N}=22)$ HIDA scans who underwent laparoscopic cholecystectomy (LC). Excellent outcomes (i.e., complete resolution or marked improvement of symptoms) were reported in both groups with no significant difference between the groups (Fuller, et al., 2000). Morris-Stiff and colleagues reported 25 patients with normal EF but recreation of symptoms upon CCK injection who underwent CCY. All patients reported complete resolution of symptoms at followup (Morris-Stiff, et al., 2011). We hypothesize that patients with normal TUS, normal HIDA scan EF, and chronic biliarylike pain will benefit from $\mathrm{CCY}$. 


\subsection{METHODS \\ $2.1 \quad$ PARTICIPANTS}

One thousand two hundred and ninety-five prospective patients who underwent elective CCY from January 2006 to December 2010 in a tertiary care institution were identified using the operating room master patient database and appropriate procedure code. Patient medical information was obtained from electronic medical records and/or clinical charts. The University of Kentucky Institutional Review Board approved all aspects of the study.

\subsection{INCLUSION/EXCLUSION CRITERIA}

Patients were excluded from further analysis if they presented with gallstones on TUS or a diagnostically abnormal HIDA scan. A HIDA scan was considered normal if the EF was at or above $35 \%$ or the radiologic interpretation was "normal." Presence or absence of reproducible pain with $\mathrm{CCK}$ injection was not considered for inclusion criteria but was noted when available. Inclusion also required chronic cholecystitis with or without the presence of gallstones on pathology. One hundred and six patients were included in the final analysis.

\subsection{DATA COLLECTION}

No direct patient contact occurred during data collection; all information was derived from medical records. Patient information was obtained that included: age, gender, race, weight, date of operation, postop clinic date, and presenting symptoms. Presenting symptoms were categorized for each patient into the presence or absence of classical biliary-like symptoms (i.e., RUQ pain, epigastric pain, postprandial pain, nausea and/or vomiting $[\mathrm{N} / \mathrm{V}]$, and nonlocalized abdominal pain). Reports for TUS, HIDA scan (including the presence or absence of reproducible pain upon CCK injection), and pathology were reviewed and results recorded. Patient outcome was determined from objective findings noted by the attending surgeon during postoperative follow-up. Complete resolution of symptoms (i.e., absence of all presenting biliary-like symptoms) and significant improvement of symptoms (i.e., reduction of presenting biliary-like symptoms to levels not affecting daily activities and not requiring additional physician consultation) were categorized as "favorable outcomes."

\subsection{DATA ANALYSIS}

Critical analysis included chi-square tests and analyses of variance calculated across groups using SPSS v.17 statistical software.

\subsection{RESULTS}

Of the 1295 patients screened for admission to the study, 106 (86 females, $82 \%, ; 20$ males, $18 \%$ ) were included in the final analysis. The mean age was $40.8 \pm$ (s.d.) 11.7 years. Patients had an average EF of $64.7 \pm$ (s.d.) 23.2\%. At postoperative follow-up, 96 patients $(90.6 \%, 79$ females) reported a favorable outcome. No patients reported treatment failure (i.e., worsening of symptoms). The average follow-up period was approximately 38 days with no 
difference between the favorable and unfavorable outcome groups. Table 1 shows patient breakdown and critical analysis with respect to outcome.

Table 1

\begin{tabular}{|l|c|c|c|}
\hline & Favorable Outcome & Unfavorable Outcome & p-value \\
\hline No. of Patients & 96 & 10 & \\
\hline Age in yrs (s.d.) & $\mathbf{4 0 . 1 ( 1 1 . 9 )}$ & $\mathbf{4 7 . 5 ( 6 . 2 )}$ & $\mathbf{0 . 0 0 5}^{\mathbf{a}}$ \\
\hline Sex (\% Male) & 17.7 & 30.0 & $0.40^{\mathrm{b}}$ \\
\hline Race (\% Minority) & 14.6 & 20.0 & $0.65^{\mathrm{b}}$ \\
\hline Mean Weight in lbs (s.d.) & $174.6(41.2)$ & $173.3(55.8)$ & $0.93^{\mathrm{a}}$ \\
\hline Mean HIDA scan EF \% (s.d.) & $63.5(23.1)$ & $77.0(20.6)$ & $0.10^{\mathrm{a}}$ \\
\hline $\begin{array}{l}\text { Postprandial } \\
\text { RUQ/Epigastric Pain (\%) }\end{array}$ & $\mathbf{7 0 . 8}$ & $\mathbf{1 0 . 0}$ & $<\mathbf{0 . 0 0 1}^{\mathbf{b}}$ \\
\hline RUQ/Epigastric pain (\%) & & & \\
\hline RUQ Pain (\%) & $\mathbf{9 5 . 9}$ & $\mathbf{6 0 . 0}$ & $\mathbf{0 . 0 0 1}^{\mathrm{b}}$ \\
\hline Epigastric Pain (\%) & $\mathbf{9 0 . 6}$ & $\mathbf{6 0 . 0}$ & $\mathbf{0 . 0 2}^{\mathbf{b}}$ \\
\hline Postprandial Pain & 12.5 & 0 & $0.60^{\mathrm{b}}$ \\
\hline Pain w/ CCK Injection (\%) & 74.0 & 50.0 & $0.14^{\mathrm{b}}$ \\
\hline Nausea/Vomiting & 64.6 & 60.0 & $0.96^{\mathrm{b}}$ \\
\hline
\end{tabular}

${ }^{\mathrm{a}} \mathrm{T}$ - test, ${ }^{\mathrm{b}}$ Fisher's Exact Test.

The mean age of the favorable and unfavorable outcome groups was 40.1 and 47.5 years, respectively $(\mathrm{p}=0.005)$. In the favorable outcome group, $70.8 \%$ presented with chief complaints that included postprandial RUQ pain and/or postprandial epigastric pain (Table 2) while only $10.0 \%$ of the unfavorable outcome group presented with the same complaint $(p<0.001)$. Differences between the groups also occurred when comparing the percentage of patients who presented with RUQ and/or epigastric pain and RUQ pain only $(\mathrm{p}=0.001$ and $\mathrm{p}=0.02$, respectively); Table 3 .

\section{Table 2}

\begin{tabular}{|c|c|c|c|c|}
\hline & \multicolumn{2}{|c|}{ Favorable Outcome } & \\
\hline & & Yes & No & \\
\hline \multirow{2}{*}{$\begin{array}{l}\text { Postprandial } \\
\text { RUQ/Epigastric Pain }\end{array}$} & Yes & 68 & 1 & PPV: $98.6 \%$ \\
\hline & No & 28 & 9 & NPV: $24.3 \%$ \\
\hline & & Sensitivity: 70.8\% & Specificity: $90.0 \%$ & \\
\hline
\end{tabular}

PPV: Positive Predictive Value, 
Table 3

\begin{tabular}{|l|l|l|l|l|}
\cline { 3 - 4 } \multicolumn{2}{c|}{} & \multicolumn{2}{c|}{ Favorable Outcome } & \multicolumn{1}{|c}{} \\
\cline { 3 - 4 } \multicolumn{2}{c|}{} & Yes & No & PPV: 93.9\% \\
\hline RUQ/Epigastric Pain & Yes & 93 & 6 & NPV: 42.9\% \\
\cline { 2 - 4 } & No & 3 & 4 & \\
\cline { 3 - 4 } & & Sensitivity: 95.9\% & Specificity: 40.0\% &
\end{tabular}

PPV: Positive Predictive Value, NPV: Negative Predictive Value

\section{DISCUSSION}

Our primary findings show that presence of chronic biliary-like pain (i.e., RUQ and/or epigastric pain associated with food) predicts short-term resolution of symptoms in patients with $\mathrm{EF}>35 \%$. Data would suggest that low EF (i.e., <35\%) is predictive of biliary dyskinesia and correlates with the presence of chronic inflammatory histological findings. This study maintains the generally accepted normal $\mathrm{EF}$ is not a reliable value for outcome prediction after CCY in patients with chronic biliary-like symptoms. Our results show that presence of chronic inflammation and favorable outcome after CCY was accurately predicted by presence of chronic biliary symptoms. These findings are concordant with other published studies (Fuller, et al., 2000; Morris-Stiff, et al., 2011).

Our study is limited in that it is retrospective in nature. Our data represent a single institution, which may affect reproducibility. Reported outcomes represented short-term follow-up and no assumptions can be deduced about long-term benefit. The size of our academic institution and status as a tertiary referral center increases the number of diverse and challenging cases. Not all HIDA scans were performed at our institution. Discrepancies in HIDA scan protocol could produce varying EF calculations (Ziessman, et al., 2006).

Since it was introduced, HIDA scanning with EF calculation has been hailed as the diagnostic procedure of choice in patients with functional gallbladder disease (Krishnamurthy, et al., 1981). It provides an anatomic and physiologic view of biliary function and predicts high success rates of CCY in patients with low EF. Conversely, mounting evidence shows that normal HIDA scan EF does not predict low success rates in patients who undergo CCY. Therefore, HIDA scan with EF calculation is omitting large populations of symptomatic patients who would potentially benefit from CCY. We recommend that an acute surgical acumen with careful assessment of patient symptomatology is the best diagnostic tool for predicting patient outcome after CCY in patients with suspected chronic acalculous cholecystitis. 


\section{REFERENCES}

Adams DB, Tarnasky PR, Hawes RH, et al. Outcome after laparoscopic cholecystectomy for chronic acalculous cholecystitis. Am Surg 1998;64:1-5; discussion 5-6. http://www.ncbi.nlm.nih.gov/pubmed/?term $=$ Adams $+\mathrm{D} \% 2 \mathrm{C}+$ Hawes $+\mathrm{RH}+\mathrm{Am}+$ Surg

Barbara L, Sama C, Morselli Labate AM, et al. A population study on the prevalence of gallstone disease: the Sirmione Study. Hepatology 1987;7:913-7. http://www.ncbi.nlm.nih.gov/pubmed/36538 $\underline{55}$

Barron LG, Rubio PA. Importance of accurate preoperative diagnosis and role of advanced laparoscopic cholecystectomy in relieving chronic acalculous cholecystitis. J Laparoendosc Surg 1995;5:357-61. http://www.ncbi.nlm.nih.gov/pubmed/?term $=$ Barron $+\mathrm{LG} \% 2 \mathrm{C}+\mathrm{Rubio}+\mathrm{PA}$

Chen PF, Nimeri A, Pham QH, et al. The clinical diagnosis of chronic acalculous cholecystitis. Surgery 2001;130:578-81, discussion 581-3. http://www.sciencedirect.com/science/article /pii/S0039606001956867

Davis GB, Berk RN, Scheible FW, et al. Cholecystokinin cholecystography, sonography, and scintigraphy: detection of chronic acalculous cholecystitis. AJR Am J Roentgenol 1982;139:1117-21. http://www.ajronline.org/doi/abs/10.2214/ajr $\underline{.139 .6 .1117}$

Fink-Bennett D, DeRidder P, Kolozsi W, et al. Cholecystokinin cholescintigraphic findings in the cystic duct syndrome. J Nucl Med 1985;26:1123-8. http://jnm.snmjournals.org/content/26/10/11 23.long

Fink-Bennett D, DeRidder P, Kolozsi WZ, et al. Cholecystokinin cholescintigraphy: detection of abnormal gallbladder motor function in patients with chronic acalculous gallbladder disease. J Nucl Med 1991;32:1695-9.

http://jnm.snmjournals.org/content/32/9/169 5.long

Fuller RA, Kuhn JA, Fisher TL, et al. Laparoscopic cholecystectomy for acalculous gallbladder disease. Proc (Bayl Univ Med Cent) 2000;13:331-3. http://www.ncbi.nlm.nih.gov/pmc/articles/P MC1312225/

Gracie WA, Ransohoff DF. The natural history of silent gallstones: the innocent gallstone is not a myth. $\mathrm{N}$ Engl $\mathrm{J}$ Med 1982;307:798-800.

http://www.nejm.org/doi/full/10.1056/NEJM $\underline{198209233071305}$

Johanning JM, Gruenberg JC. The changing face of cholecystectomy. Am Surg 1998;64:643-7, discussion 647-8. http://www.ncbi.nlm.nih.gov/pubmed/96552 $\underline{75}$

Krishnamurthy GT, Bobba VR, Kingston E. Radionuclide ejection fraction: a technique for quantitative analysis of motor function of the human gallbladder. Gastroenterology 1981;80:482-90. 
http://www.ncbi.nlm.nih.gov/pubmed/74504 $\underline{41}$

Majeski J. Gallbladder ejection fraction: an accurate evaluation of symptomatic acalculous gallbladder disease. Int Surg 2003;88:95-9.

http://www.ncbi.nlm.nih.gov/pubmed/12872 $\underline{903}$

Middleton GW, Williams JH. Diagnostic accuracy of 99Tcm-HIDA with cholecystokinin and gallbladder ejection fraction in acalculous gallbladder disease. Nucl Med Commun 2001;22:657-61. http://www.ncbi.nlm.nih.gov/pubmed/11403 $\underline{177}$

Misra DC Jr, Blossom GB, Fink-Bennett D, et al. Results of surgical therapy for biliary dyskinesia. Arch Surg 1991;126:957-60. http://archsurg.jamanetwork.com/article.asp $\underline{\mathrm{x} \text { ?articleid }=595139}$

Morris-Stiff G, Falk G, Kraynak L, et al. The cholecystokin provocation HIDA test: recreation of symptoms is superior to ejection fraction in predicting medium-term outcomes. J Gastrointest Surg 2011;15:3459.

http://link.springer.com/article/10.1007\%2Fs 11605-010-1342-4

Pickleman J, Peiss RL, Henkin R, et al. The role of sincalide cholescintigraphy in the evaluation of patients with acalculus gallbladder disease. Arch Surg 1985;120:693-7. .

http://archsurg.jamanetwork.com/article.asp $\underline{\mathrm{x} \text { ?articleid }=591054}$
Poynter MT, Saba AK, Evans RA. Chronic acalculous biliary disease: cholecystokinin cholescintigraphy is useful in formulating treatment strategy and predicting success after cholecystectomy. Am Surg 2002;68:382-4.

http://www.ncbi.nlm.nih.gov/pubmed/?term =Poynter+MT\%2C+Saba+AK\%2C+Evans+ $\underline{\mathrm{RA}}$

Ross M, Brown M, McLaughlin K, et al. Emergency physician-performed ultrasound to diagnose cholelithiasis: a systematic review. Acad Emerg Med 2011;18:227-35. http://onlinelibrary.wiley.com/doi/10.1111/j. $1553-$

2712.2011.01012.x/abstract;jsessionid=DF4 F49FC2E79192625178A6A0B871811.f04t0 $\underline{1}$

Topper TE, Ryerson TW, Nora PF. Quantitative gallbladder imaging following cholecystokinin. J Nucl Med 1980;21:694-6. http://jnm.snmjournals.org/content/21/7/694. $\underline{\text { long }}$

Yap L, Wycherley AG, Morphett AD, et al. Acalculous biliary pain: cholecystectomy alleviates symptoms in patients with abnormal cholescintigraphy. Gastroenterology 1991;101:785-93. http://www.ncbi.nlm.nih.gov/pubmed/?term $=\mathrm{Yap}+\mathrm{L} \% 2 \mathrm{C}+\mathrm{W}$ ycherley $+\mathrm{AG} \% 2 \mathrm{C}+$ Morphe $\underline{\mathrm{tt}+\mathrm{AD}}$

Yost F, Margenthaler J, Presti M, et al. Cholecystectomy is an effective treatment for biliary dyskinesia. Am J Surg 1999;178:462-5. 
http://www.sciencedirect.com/science/article /pii/S0002961099002287

Zech ER, Simmons LB, Kendrick RR, et al. Cholecystokinin enhanced hepatobiliary scanning with ejection fraction calculation as an indicator of disease of the gallbladder. Surg Gynecol Obstet 1991;172:21-4. http://www.ncbi.nlm.nih.gov/pubmed/?term $=\mathrm{Zech}+\mathrm{ER} \% 2 \mathrm{C}+\mathrm{Simmons}+\mathrm{LB} \% 2 \mathrm{CKendric}$ $\underline{\mathrm{k}+\mathrm{RR}}$

Ziessman HA. Functional hepatobiliary disease: chronic acalculous gallbladder and chronic acalculous biliary disease. Semin Nucl Med 2006;36:119-32. http://www.sciencedirect.com/science/article /pii/S000129980500070X 